\title{
DIMENSION OF METRIC SPACES AND HILBERT'S PROBLEM $13^{1}$
}

BY PHILLIP A. OSTRAND

Communicated by R. P. Boas, March 10, 1965

In 1957 A. N. Kolmogorov [1] and V. I. Arnol'd [2] obtained the following result (answering Hilbert's conjecture in the negative):

THEOREM. For every integer $n \geqq 2$ there exist continuous real functions $\psi^{p q}$, for $p=1,2, \cdots, n$ and $q=1,2, \cdots, 2 n+1$, defined on the unit interval $E^{1}=[0,1]$, such that every continuous real function $f$, defined on the $n$-dimensional unit cube $E^{n}$, is representable in the form

$$
f\left(x_{1}, \cdots, x_{n}\right)=\sum_{q=1}^{2 n+1} x_{q}\left[\sum_{p=1}^{n} \psi^{p q}\left(x_{p}\right)\right],
$$

where the functions $\chi_{q}$ are real and continuous.

The proof of the theorem relies on two properties of $E^{1}$, namely, $E^{1}$ is compact and of dimension 1. (By dimension we shall always mean covering dimension.) This paper generalizes the work of Kolmogorov and Arnol'd to obtain the following result:

Theorem 2. For $p=1,2, \cdots, m$ let $X^{p}$ be a compact metric space of finite dimension $d p$, and let $n=\sum_{p=1}^{n} d_{p}$. There exist continuous functions $\psi^{p q}: X^{p} \rightarrow[0,1]$, for $p=1, \cdots, m$ and $q=1,2, \cdots, 2 n+1$, such that every continuous real function $f$ defined on $\prod_{p=1}^{m} X^{p}$ is representable in the form

$$
f\left(x_{1}, \cdots, x_{m}\right)=\sum_{q=1}^{2 n+1} \chi_{q}\left[\sum_{p=1}^{m} \psi^{p q}\left(x_{p}\right)\right],
$$

where the functions $\chi_{q}$ are real and continuous.

The proof of Theorem 2 makes use of the following new character1zation of dimension of metric spaces which is of interest in itself.

Theorem 1. A metric space $X$ is of dimension $\leqq n$ if and only if for each open cover $\mathfrak{C}$ of $X$ and each integer $k \geqq n+1$ there exist $k$ discrete families of open sets $\mathfrak{u}_{1}, \cdots, \mathfrak{u}_{k}$ such that the union of any $n+1$ of the $\mathfrak{u}_{i}$ is a cover of $X$ which refines $\mathfrak{e}$.

1 This research was partially supported by a National Science Foundation Fellow* ship. 
By a discrete family of sets we mean a family such that each point has a neighborhood which meets at most one member of the family. For dimension 0 Theorem 1 reduces to the following result, which is well known: A metric space $X$ is of dimension $\leqq 0$ if and only if each open cover of $X$ may be refined by a discrete family of open sets.

This note presents brief proofs of Theorems 1 and 2 .

Proof of Theorem 1. It suffices to show that if $X$ is of dimension $\leqq n$, then for each open cover $\mathcal{e}$ of $X$ and each integer $k \geqq n+1$ there exist $k$ discrete families of open sets $\mathfrak{u}_{1}, \cdots, \mathfrak{u}_{k}$ which refine $\mathcal{C}$, any $n+1$ of which cover $X$. We prove this by induction on $k$. Let $X$ be of dimension $\leqq n$ and let $\mathfrak{e}=\left\{C_{\alpha} ; \alpha \in I\right\}$ be an open cover of $X$. By passing to a refinement if necessary, we may suppose that $\mathfrak{C}$ is locally finite. We may write $X$ as $X=\cup_{i=1}^{n+1} X_{i}$ where each $X_{i}$ is a subspace of dimension 0 .

Let $\mathfrak{e}_{i}=\left\{C_{\alpha} \cap X_{i}: \alpha \in I\right\} . \mathfrak{e}_{i}$ is an $X_{i}$-open cover of $X_{i}$. By the case of dimension 0 there exists for each $i$ a disjoint family $v_{i}$ of $X_{i}$-open sets which covers $X_{i}$ and refines $\mathfrak{C}_{i}$.

Let $I$ be well ordered by $<$. For each $\alpha \in I$ and $i=1, \cdots, n+1$, let $W_{\alpha}^{i}=U\left\{V \in \mathcal{V}_{i}: V \subset C_{\alpha} \cap X_{i}\right.$ and for each $\left.\beta<\alpha, V \nsubseteq C_{\beta} \cap X_{i}\right\}$, and let $W_{i}=\left\{W_{\alpha}^{i}: \alpha \in I\right\}$. Distinct members of $W_{i}$ are disjoint.

Let $Z_{\alpha}^{i}=\left\{x \in C_{\alpha}: d\left(x, W_{\alpha}^{i}\right)<d\left(x, \cup_{\beta<\alpha} W_{\beta}^{i}\right)\right\}$ and let $Z_{i}=\left\{Z_{\alpha}^{i}: \alpha \in I\right\}$. $W_{\alpha}^{i} \subset Z_{\alpha}^{i} \subset C_{\alpha}$ for each $i$ and $\alpha$. Each $Z_{i}$ is a locally finite family of disjoint open sets of $X$ which refines $\mathcal{C}$ and covers $X_{i}$. It follows that $U_{i=1}^{n+1} Z_{i}$ covers $X$.

As before, there exist closed sets $D_{\alpha}^{i} \subset Z_{\alpha}^{i}$ such that $\left\{D_{\alpha}^{i}: \alpha \in I\right.$; $i=1, \cdots, n+1\}$ covers $X$. Choose open sets $U_{\alpha}^{i}$ such that $D_{\alpha}^{i} \subset U_{\alpha}^{i}$ $\subset \mathrm{Cl}\left(U_{\alpha}^{i}\right) \subset Z_{\alpha}^{i}$, and let $\mathfrak{u}_{i}=\left\{U_{\alpha}^{i}: \alpha \in I\right\}$. Each $\mathfrak{u}_{i}$ is discrete and refines $\mathcal{e}$, and $\mathfrak{u}_{1}, \cdots, \mathfrak{u}_{n+1} \operatorname{cover} X$.

Suppose now that $k>n+1$ and that $\mathfrak{u}_{1}, \cdots, \mathfrak{u}_{k-1}$ are discrete families of open sets which refine $\mathfrak{e}$, any $n+1$ of which cover $X$. We will construct a subset $A$ of $X$ and a discrete family $\mathfrak{U}_{k}$ of open sets which refines $\mathfrak{C}$, such that any $n$ of the families $\mathfrak{u}_{1}, \cdots, \mathfrak{u}_{k-1}$ cover $X-A$ and $\mathfrak{u}_{k}$ covers $A$.

Let $\mathfrak{A}=\left\{\boldsymbol{\gamma}=\left(\gamma_{1}, \cdots, \gamma_{n}\right): 1 \leqq \gamma_{1}<\gamma_{2}<\cdots<\gamma_{n} \leqq k-1\right\}$. For $\gamma \in \mathfrak{A}$, let $A_{\gamma}=\cap_{i=1}^{n}\left(X-\cup \mathfrak{u}_{\gamma_{i}}\right)$, and $A=\bigcup_{\gamma \in \mathfrak{A}} A_{\gamma}$. Each $A_{\gamma}$ is closed and $A_{\gamma} \cap A_{\delta}=\varnothing$ for $\gamma \neq \delta$. Hence there exist open sets $B_{\gamma}$ such that $A_{\gamma} \subset B_{\gamma}$ and $\mathrm{Cl}\left(B_{\gamma}\right) \cap \mathrm{Cl}\left(B_{\delta}\right)=\varnothing$ for $\gamma \neq \delta$. For $\gamma \in \mathfrak{A}$, there exists a positive integer $j_{\gamma} \leqq k-1$ such that $j_{\gamma} \notin\left\{\gamma_{i}: i=1,2, \cdots, n\right\} . u_{j_{\gamma}}$ covers $A_{\gamma}$. Let $\mathfrak{u}_{k}=\left\{U \cap B_{\gamma}: \gamma \in \mathfrak{A}\right.$ and $\left.U \in \mathfrak{U}_{j_{\gamma}}\right\}$. $\mathfrak{u}_{k}$ is a discrete family of open sets which covers $A$ and refines $\mathrm{C}$. Thus the proof of Theorem 1 is complete.

Proof of Theorem 2. For each integer $p, p=1, \cdots, m$, each 
integer $q, q=1,2, \cdots, 2 n+1$, and each integer $k, k=1,2, \cdots$, there exist positive real numbers $\gamma_{k}$ and $\epsilon_{k}$, distinct positive prime numbers $r_{k}^{p q}$, discrete families $S_{k}^{p q}$ of open sets of $X^{p}$, and continuous functions $f_{k}^{p g}: X^{p} \rightarrow[0,1]$ such that:

(1) $\lim _{k \rightarrow \infty} \gamma_{k}=\lim _{k \rightarrow \infty} \epsilon_{k}=0$;

(2) each member of $S_{k}^{p d}$ is of diameter $\leqq \gamma_{k}$ and for each fixed $p$ and $k$ any $d_{p}+1$ of the families $S_{k}^{p q}$ cover $X^{p}$;

(3) $m \epsilon_{k}<1 / \prod_{p=1}^{m} r_{k}^{p q}$ for each $q=1,2, \cdots, 2 n+1$;

(4) $f_{k}^{p q}$ is constant on each member of $S_{k}^{p q}$, the constant being an integral multiple of $1 / r_{k}^{p q}$, and takes different values on distinct members of $S_{k}^{z q}$;

(5) For each $j<k$ and $x \in X^{p}, f_{j}^{p q}(x) \leqq f_{k}^{p q}(x) \leqq f_{j}^{p q}(x)+\epsilon_{j}-\epsilon_{k}$.

The $\gamma_{k}, \epsilon_{k}, r_{\mathbf{k}}^{p q}, s_{\boldsymbol{k}}^{p q}$, and $f_{\boldsymbol{k}}^{p q}$ are defined inductively on $k$.

Let $X=\prod_{p=1}^{m} X^{p} . X$ is a metric space with metric

$$
d\left(\left(x_{1}, \cdots, x_{m}\right),\left(y_{1}, \cdots, y_{m}\right)\right)=\sum_{p=1}^{m} d\left(x_{p}, y_{p}\right) .
$$

For each $q$ and $k$ let $J_{k}^{q}=\left\{\prod_{p=1}^{m} C^{p}: C^{p} \in s_{\mathbf{k}}^{p q}\right.$ for each $\left.p\right\}$. Each $\Im_{\mathbf{k}}^{q}$ is a discrete family of open sets of $X$, and each member of $J_{k}^{q}$ is of diameter $\leqq m \gamma_{k}$. For each $k$ any $n+1$ of the families $J_{k}^{q}$ cover $X$.

Let $\psi^{p q}(x)=\lim _{k \rightarrow \infty} f_{k}^{p q}(x)$ for $x \in X^{p}$. For each $k$ and each $x \in X^{p}$, $f_{k}^{p q}(x) \leqq \psi^{p q}(x) \leqq f_{k}^{p q}(x)+\epsilon_{k}$. Thus $\psi^{p q}$, being the uniform limit of the $f_{k}^{p q}$, is continuous.

Let $\phi^{q}\left(x_{1}, \cdots, x_{m}\right)=\sum_{p=1}^{m} \psi^{p q}\left(x_{p}\right)$ for $\left(x_{1}, \cdots, x_{m}\right) \in X$. Let $u_{\mathbf{k}}^{q}=\left\{\phi^{q}(C): C \in J_{\underline{k}}^{q}\right\}$. If $C=\prod_{p=1}^{m} C^{p} \in J_{\vec{k}}^{q}$, then $\phi^{q}(C)$ is contained in the interval $\left[\sum_{p=1}^{m} f_{k}^{p q}\left(C^{p}\right), \sum_{p=1}^{m} f_{k}^{p q}\left(C^{p}\right)+m \epsilon_{k}\right]$. By condition (3) these closed intervals are disjoint for each fixed $q$ and $k$. Hence each $\mathcal{U}_{\mathrm{k}}^{q}$ is discrete.

Let $f$ be a continuous real-valued function on $X$. For each integer $r \geqq 0$ and $q=1, \cdots, 2 n+1$ there exists a positive integer $k_{r}$ and continuous functions $\chi_{r}^{q}: R \rightarrow R$ ( $R$ denotes the real line, $k_{0}=1$ and $\chi_{0}^{q}=0$ for each $q$ ) such that if $f_{r}(x)=\sum_{q=1}^{2 n+1} \sum_{s=0}^{r} \chi_{s}^{q}\left(\phi^{q}(x)\right)$ for $x \in X$ and if $M_{r}=\sup _{x \in X}\left|\left(f-f_{r}\right)(x)\right|$, then:

(6) $k_{r+1}>k_{r}$;

(7) if $d(a, b)<m \gamma_{k_{r+1}}$, then $\left|\left(f-f_{r}\right)(a)-\left(f-f_{r}\right)(b)\right|<(2 n+2)^{-1} M_{r}$;

(8) $\chi_{r+1}^{q}$ is constant on each member of $\mathcal{u}_{\hat{k}_{r+1}^{q}}^{q}$, its value on $\phi^{q}(C)$ for $C \in J_{k_{r+1}}^{g}$ being $(n+1)^{-1}\left(f-f_{r}\right)(y)$ for some arbitrarily chosen element $y$ of $C$;

(9) $\left|\chi_{r+1}^{q}(a)\right| \leqq(n+1)^{-1} M_{r}$ for each $a \in R$.

The $k_{r}$ and $\chi_{r}^{q}$ are defined inductively on $r$. It is easily deduced from (7) and (8) that 
(10) $\left|(n+1)^{-1}\left(f-f_{r}\right)(x)-\chi_{r+1}^{q}\left(\phi^{q}(x)\right)\right|<(n+1)^{-1}(2 n+2)^{-1} M_{r}$ for $x \in \bigcup\left\{T: T \in J_{\mathfrak{k}_{r+1}}^{Q}\right\}$.

For each $x \in X$ there are at least $n+1$ distinct values of $q$ such that that $x \in \bigcup\left\{T: T \in J_{\mathbf{k}_{r+1}}^{q}\right\}$. Adding (10) for $n+1$ values of $q$ and (9) for the other $n$ values of $q$ yields

$$
\left|\left(f-f_{r+1}\right)(x)\right|=\left|\left(f-f_{r}\right)(x)-\sum_{q=1}^{2 n+1} \chi_{r+1}^{q}\left(\phi^{q}(x)\right)\right|<\frac{2 n+1}{2 n+2} M_{r} .
$$

Then $M_{r+1}<(2 n+1)(2 n+2)^{-1} M_{r}$, so $M_{r}<\left((2 n+1)(2 n+2)^{-1}\right)^{r} M_{0}$ for each $r$ and $\lim _{r \rightarrow \infty} M_{r}=0$. Hence $f(x)=\lim _{r \rightarrow \infty} f_{r}(x)$ for all $x \in X$. Moreover, by condition (9) the functions $\sum_{s=0}^{n} \chi_{s}^{q}$ converge uniformly for each $q$ to a continuous function $\chi^{q}: R \rightarrow R$ and

$$
f(x)=\lim _{r \rightarrow \infty} f_{r}(x)=\lim _{r \rightarrow \infty} \sum_{q=1}^{2 n+1} \sum_{s=0}^{r} \chi_{s}^{q}\left(\phi^{q}(x)\right)=\sum_{q=1}^{2 n+1} \chi^{q}\left(\phi^{q}(x)\right) .
$$

This completes the proof of Theorem 2 .

\section{REFERENCES}

1. A. N. Kolmogorov, Representation of functions of many variables, Dokl. Akad. Nauk 114 (1957), 953-956; English transl., Amer. Math. Soc. Transl. (2) 17 (1961), 369-373.

2. V. I. Arnol'd, On functions of three variables, Dokl. Akad. Nauk 114 (1957), 679-681; English transl., Amer. Math. Soc. Transl. (2) 28 (1963), 51-54.

NORTHWESTERN UNIVERSITY 